\title{
Importance of human papillomavirus vaccination in prevention of cancers among HIV/AIDS patients
}

\author{
Mostafa Dianatinasab ${ }^{1,2}$, Hassan Joulaei ${ }^{3}$, Mohammad Fararouei $^{1}$ \\ ${ }^{1}$ Shiraz HIV/AIDS Research Center, Institute of Health, Shiraz University of Medical Sciences, Shiraz, Iran \\ ${ }^{2}$ Department of Epidemiology, Faculty of Public Health, Shiraz University of Medical Sciences, Shiraz, Iran \\ ${ }^{3}$ Health policy Research Center, Institute of Health, Shiraz University of Medical Sciences, Shiraz, Iran
}

Human papillomavirus (HPV) is recognized as one of the major infectious causes of several types of cancer worldwide. For example, according to the International Agency for Research on Cancer (IARC), strong evidence exist for a causal association between HPV infection and cancers of the uterine cervix, penis, vulva, vagina, anus, and oropharynx [1]. Immunocompromised populations are known to have an increased risk of HPV-associated cancers. Human immunodeficiency virus (HIV)-infected individuals form the largest immunocompromised population with special importance from a public health prospect [2]. A large number of individuals infected with HIV are also infected with HPV [3]. There has been increasing understanding of the HPV-HIV co-infection leading to high mortality among the patients [1].

Linkage studies on HIV/acquired immune deficiency syndrome (AIDS) and cancer registries have shown about a 20-fold increase in cervical cancer among HIV-positive women compared to HIV-negative women. In addition, it has been shown that resistance to treatment of HPV-related diseases is stronger with immunocompromise seen in HIV-positive individuals, among which, faster development of HPV-associated cancers is seen [4]. Furthermore, cohort studies provided some hard evidences that the risk of acquiring cervical HPV infection is higher among HIVpositive than HIV-negative women [5]. Among HIV-infected people, risk of cancer increases because of immunosuppression. Among all types of cancer, HPV is more strongly associated with cervical and anal cancers [6]. The association is stronger when the function of immune system diminishes, as in HIV patients.

Address for correspondence: Mostafa Dianatinasab, MSc, Shiraz HIV/AIDS Research Center, Institute of Health, Shiraz, University of Medical Sciences, Shiraz, Iran, phone: +989369390714, e-mail: dianati.epid@gmail.com

There seem to be differences in the type of HPV and the effects of host immune status on the risk of HPV infection. Most notably among all types of HPV, prevalence of HPV-16, that by itself accounts for approximately half of all cervical cancers, was found to be least affected by CD4 count. This has been interpreted as a conclusive evidence that HPV-16 may, in part, be the dominant cause of cervical cancer because of an innate ability to thrive in people with competent immune system. Consequently, HIV-associated immunosuppression may have less impact on HPV-16 than other HPV subtypes. Like cervical cancer, anal cancer is also associated with HPV infection. As a matter of fact, HPV16 is even more common in anal than in cervical cancer, whereas HPV-18 and other oncogenic HPV types are found in only a subset of anal tumors [7]. Meta-analysis studies have shown that anal cancer incidence is 30 times higher in HIV-infected individuals compared to the general population [8]. In addition, oropharyngeal cancer occurs at higher rates in HIV-positive men and women than in the general population [9]. Along with this increased risk of oropharyngeal cancers, HIV-infected individuals are also at higher risk of oral HPV infection [9].

Observational studies have shown that HIV-positive women can mount a humoral immune response against re-infection or possible reactivation of latent infection with $\mathrm{HPV}$, and it is reasonable to assume that vaccination, which leads to titers well above those of natural infection, would induce protective HPV antibody titers in at least a significant portion of HIV-positive patients [10-12]. HPV antibodies should bind to the virus regardless of whether it is a first or a subsequent exposure, and therefore high antibody titers

Article history:

Received: 30.09.2017

Received in revised form: 20.01.2018

Accepted: 12.02.2018

Available online: 15.08.2018
International Journal of HIV-Related Problems

HIV \& AIDS

R e vi e w 
may offer some protection against re-infection or possible reactivation of $\mathrm{HPV}$ infection.

It is suggested that HPV vaccination is possibly more effective if given to an HIV-infected individual when the person's immune system is less compromised. These include: (a) prior to an advanced stage of HIV/AIDS, (b) after initiation of successful highly active antiretroviral therapy (HAART), (c) prior to the initiation of transplant and/or iatrogenic immune suppression, or (d) at the time of reduced iatrogenic immunosuppression in transplant recipients [9].

Overall, given the high rates of HPV-infection and the HPV associated cancers in immunocompromised individuals, there is sufficient reason to consider HPV vaccination in those groups. However, vaccination strategies need to be based on the outcome of clinical trials in these populations. Ideally, vaccine trials on immunocompromised women and men should concurrently assess cervical, anal, and oral HPV infection. In this respect, penile and vulvar infections should be assessed as well. The association of HPV-16 with anal and oropharyngeal cancers provides an additional reason for exploring the efficacy of HPV vaccination in men, beyond the goal of reducing transmission of HPV-16 to women [9].

It is suggested that vaccination of immune-suppressed individuals may be the best long-term strategy against the increasing risk of some types of cancer [13], particularly cervical, anal, and oral cancers $[1,9]$. This strategy is almost well-recognized in developed countries. For instance, there are guidelines, which provide recommendations on vaccination in cancer patients, HIV patients, and all other immunocompromised [14].

However, there is paucity of data for HPV vaccination among HIV-infected individuals in less developed countries, where HIV/AIDS remains a persistent real problem [15]. Hence, further investigation with more robust data sets is essential in these countries.

\section{Conflict of interest}

The authors declare no potential conflicts of interest with respect to the research, authorship, and/or publication of this article.

\section{References}

1. Bosch FX, Broker TR, Forman D, et al. Comprehensive control of human papillomavirus infections and related diseases. Vaccine 2013; 31: H1-H31.

2. Petrosky E, Bocchini Jr JA, Hariri S, et al. Use of 9-valent human papillomavirus (HPV) vaccine: updated HPV vaccination recommendations of the advisory committee on immunization practices. MMWR Morb Mortal Wkly Rep 2015; 64: 300-304.

3. Denny LA, Franceschi S, de Sanjosé S, et al. Human papillomavirus, human immunodeficiency virus and immunosuppression. Vaccine 2012; 30: F168-F174.

4. Reusser NM, Downing C, Guidry J, Tyring SK. HPV Carcinomas in Immunocompromised Patients. J Clin Med 2015; 4: 260-281.

5. De Vuyst H, Lillo F, Broutet N, Smith JS. HIV, human papillomavirus, and cervical neoplasia and cancer in the era of highly active antiretroviral therapy. Eur J Cancer Prevent 2008; 17: 545-554.
6. Malagón T, Franco EL. Human Papillomavirus Vaccination: Making Sense of the Public Controversy. In: Recurrent Respiratory Papillomatosis. Campisi P (ed.). Springer, 2018; 59-94.

7. Franco EL, de Sanjosé S, Broker TR, et al. Human papillomavirus and cancer prevention: Gaps in knowledge and prospects for research, policy, and advocacy. Vaccine 2012; 30: F175-F182.

8. Silverberg MJ, Lau B, Justice AC, et al. Risk of anal cancer in HIV-infected and HIV-uninfected individuals in North America. Clin Infect Dis 2012; 54: 1026-1034.

9. Palefsky JM, Gillison ML, Strickler HD. HPV vaccines in immunocompromised women and men. Vaccine 2006; 24: S140-S146.

10. Lenz P, Lowy DR, Schiller JT. Papillomavirus virus-like particles induce cytokines characteristic of innate immune responses in plasmacytoid dendritic cells. Eur J Immunol 2005; 35: 1548-1556.

11. Palefsky JM, Gillison ML, Strickler HD. Chapter 16: HPV vaccines in immunocompromised women and men. Vaccine 2006; 24 (Suppl 3): 140-146.

12. Lin C, Franceschi S, Clifford GM. Human papillomavirus types from infection to cancer in the anus, according to sex and HIV status: a systematic review and meta-analysis. Lancet Infect Dis 2018; 18: $198-206$

13. Lowy DR, Schiller JT. Reducing HPV-associated cancer globally. Cancer Prev Res (Phila) 2012; 5: 18-23.

14. Lopez A, Mariette X, Bachelez H, et al. Vaccination recommendations for the adult immunosuppressed patient: A systematic review and comprehensive field synopsis. J Autoimmun 2017; 80: 10-27.

15. Joulaei H, Shooshtarian S, Dianatinasab M. Is UNAIDS 90-90-90 target a Dream or a Reality for Middle East and North Africa Region on Ending the AIDS Epidemic? A Review Study. AIDS Rev 2018; 20 : 83-93. 